\title{
Technical Database on Robotics-based Educational Platforms for K-12 students
}

\author{
Hugo Costelha \\ IPLeiria, Leiria, Portugal \\ INESC TEC, Porto Portugal \\ hugo.costelha@ipleiria.pt
}

\author{
Carlos Neves \\ IPLeiria, Leiria, Portugal \\ INESCC, Coimbra, Portugal \\ carlos.neves@ipleiria.pt
}

\begin{abstract}
Educational robotics has had an increasing growth in the past years, mainly in teaching Science, Technology, Engineering, Arts and Mathematics (STEAM). These roboticsbased learning methods have since gone from home to be used every day in school learning activities. There still is, however, a big moat from the available resources and the effective use of these tools by teachers in K-12 schools. This study aims to gather in a single location a dataset of most available educational robotic platforms and related learning materials. The goal is to have this knowledge open, freely accessible and editable by manufactures and learning resources providers, helping to increase the adoption of educational robotics in STEAM education.
\end{abstract}

Keywords—STEAM; educational robotics; learning resources; database.

\section{INTRODUCTION}

Learning and using Computer Science tools from early ages is now a standard part of K-12 students' curricula (form primary school to high/secondary school students) both in developed countries [1] and developing countries [2]. Europe itself puts great importance on the use of these tools for teaching Science, Technology, Engineering and Mathematics (STEM) [3].

In most cases, particularly when these tools were first introduced in the curricula of young students, the focus was mainly in developing reasoning through computational thinking, as described in [4], using computers or tablets. Since then, most educational programs have evolved by including robots [5] [6], with positive results [7]. In fact, there are many studies on the use of robots to foster and improve learning [8] [9], starting from 1980 with the TURTLE/LOGO platform [10]. However, as described in [8], activities involving robots are usually not integrated into classroom activities, but occur mostly as extracurricular activities, outside of standard class time, where activities and experiments are supervised by tutors.

As described in [9], educational robotics allows students, on a first stage, to learn programming and reasoning, either through textual programming, visual programming, or with other software tools. The advantage of using robots is that students can see concepts physically and learn in other areas, such as building physical systems [11] and learning electrical and electronics related concepts, helping shift the students from "consumers" to "makers" [12]. On the other hand, these advantages are the main reasons why learning with robotics is usually not done within the classroom. Having teachers with qualifications in these domains is not usual and requires proper training, leading often to the tutor-based extra-curricular activities described earlier [8]. As stated in [8], although some studies have shown no learning improvement when using robotics, most studies show a learning enhancement through the use of robotics in education [13]. Furthermore, it has also been shown that teachers also have higher engagement when involved in these activities [14].

There are currently many software and hardware platforms with related materials and educational activities using robots. These cover technical and non-technical skills, such as social skills, reasoning, mathematics, physics, engineering, among others (STEAM, i.e., STEM plus Arts). Determining which robotic platforms and materials are available is a daunting task, given the growth of educational robotics, particularly for teachers with low technical background (K-12 teachers).

There are few articles which provide a review on available educational robotic platforms [15] and coding materials [16], but not many details are given and the information is limited to the articles timeframe. In terms of websites these are also scarce, with examples as Wikipedia ${ }^{1}$, which includes a table summarizing some of the available educational robotic platforms, or robots 4 schools $^{2}$, which works as a hub for educational robotics in Switzerland, aggregating information on some educational robotic platforms and learning/teaching materials as shown in Fig. 1 (here one can see the school grade where each educational robot can be used, where Electronics means platforms implying electronics learning).

\begin{tabular}{|c|c|c|c|c|c|c|c|cc|cc|c|c|}
\hline Year & $\mathbf{1}$ & $\mathbf{2}$ & $\mathbf{3}$ & $\mathbf{4}$ & $\mathbf{5}$ & $\mathbf{6}$ & $\mathbf{7}$ & $\mathbf{8}$ & $\mathbf{9}$ & $\mathbf{1 0}$ & $\mathbf{1 1}$ & $\mathbf{1 2}$ \\
Age & $\mathbf{4}$ & $\mathbf{5}$ & $\mathbf{6}$ & $\mathbf{7}$ & $\mathbf{8}$ & $\mathbf{9}$ & $\mathbf{1 0}$ & $\mathbf{1 1}$ & $\mathbf{1 2}$ & $\mathbf{1 3}$ & $\mathbf{1 4}$ & $\mathbf{1 5}$ \\
\hline BeeBot & & & & & & & & & & & & \\
\hline WeDo & & & & & & & & & & & & \\
\hline Thymio II & & & & & & & & & & & & \\
\hline Mindstorms & & & & & & & & & & & & \\
\hline Electronics & & & & & & & & & & & & \\
\hline
\end{tabular}

Fig. 1-Grades where each robot can be used for education ${ }^{2}$.

One key aspect of this type of learning is not to follow a completely specified learning path so, many different materials exist, even for a given country and curricular program. Considering this and the need to provide for an easier access to the available educational robotic platforms and materials, we started this work.

This work focuses on creating one central database with a web-based interface which provides an easy to access list of available robots and corresponding learning materials, allowing searching by type, school year, spoken language, programming 
environment, among others. The idea is not to provide the materials per se, but links to the pages that host those materials. To describe the developed work, this paper is structured as follows: section II describes the hardware platforms included in the study and the various points being compared among them; section III provides for a similar approach for the available software and learning resources; section IV describes the conclusions and future work.

\section{HARDWARE PlatForms FOR EDUCATIONAL RoBOTICS}

There are many robotic platforms developed for education, mainly in STEM, with high prospects [15] [17] and with class related learning materials or learning materials that can be easily adapted for a class. This study focuses on commercially available platforms or platforms which have available all the needed documentation to reproduce the robots using commercially available components. At this stage of the study, we also opt to consider only robots with a small number of DoF, considering K-12 grades, which results mainly in wheel-based or simple humanoid robots. Robots that had complex AI skills but lack the low programming approach, or which do not allow students and teachers to understand most of what is running on the robot, were also not considered. The low cost needed to allow cheaper acquisition/build of educational robots is also an important factor, so only robots below $250 €$ or kits (allowing multiple robots) below $500 €$ were included. Robotic arms were also not included at this stage, given that the mobility associated with mobile robots is usually more appealing to young students, and given the higher availability of learning materials for mobile educational robots. The project is at a starting phase, with the expectation that manufacturers and material providers contribute to maintain the information correct and up to date.

Table 1 summarizes the information collected so far. Given the amount of gathered information, not all studied robots are shown and not all characteristics were included (see the full list at the project $w_{e b s i t e}{ }^{3}$. Table 1 includes, from left to right:

- Project: name and link of the robotic platform ${ }^{4}$;

- Ages: applicable ages. Note that in-class ages can be lower, given the teachers' support;

- Assembly Type: can be RKA (Raw Kit Assembly), which implies assembly and soldering; KA (Kit Assembly), which implies assembly; or AP (Assembled Platform), which comes pre-assembled;

- Motion type: can be wheeled, mainly 2W (2 Wheels); legged, mainly 2L (2 Legs); or configurable (C), meaning that several different motion models can be assembled;

- Open-source: whether the platform is fully open-source (mechanics, electronics and software), has some opensource parts, or is closed-source;

- 3D-print: whether the robot structure and other addon parts can be created using a 3D printer;

- Text-based Programming Languages (TPL): available text-based programing languages;
- Visual Programming Languages (VPL): available visual (usually block-based) programming languages;

- Wireless communications: available wireless communication protocols. When available, these allow user-to-robot or robot-to-robot wireless communications;

- Programming upload: type of program uploads available. If wireless upload is possible, it allows for a friendlier experience and increased connectors lifespan;

- Sensors: included (or additional) sensors. In the case of sound recording/playback, doing so through a (wirelessly) connected app was also considered;

- Actuators: included (or additional) actuators;

- Brick compatibility: being compatible with brick-type systems, like LEGO, for instance, allows the students to easily expand their constructions;

- Learning materials: quality/quantity of learning materials enabling the user (mainly the teacher in this case) to learn how to use the robotic platform:

+: no specific materials exist, but there are available materials that can be adapted;

++ : learning materials exist, but are not extensive;

+++ : extensive set of leaning materials exist, covering all the robot functions through several examples.

- Classroom materials: quality/quantity of classroom materials, which can be used by teachers in their classes:

No: no classroom specific materials exist;

+ : Classroom materials exist, but are not grade-organized or field-organized;

++ : Classroom materials exist, but are organized by fields or, if grade-organized, if includes few activities;

+++ : Classroom activities exist, are extensive and are grade-organized or, if field-organized, it is clearly defined and provides for a large set of activities;

- Use: this field describes the ease of use:

-: Soldering and complex assembly required. Difficult to use, implying the setup of operating systems and complex software in the robot;

+: Soldering and complex assembly required. Mostly text-based programming approach, with no high-level wireless interface;

++: High-level block-based interface for programming, possibly with onboard buttons to aid operations;

+++: Very easy high-level wireless operation available with block-based programming. Additional add-ons easy to set up;

- Cost: the cost is mostly indicative and based on the time of publish, considering Europe-based shops. 
Some manufacturers provide more than one robot. In those cases where the differences were not considerable, only one robot was chosen (for space reasons). For instance, the Anprino was developed by ANPRI, a Portuguese computed science teachers' association, and is available also in two other models, the Anprino Luís, with an infrared line sensor, and Anprina Nandy, with an ultrasound sensor, both leaving out the Bluetooth model. In the Thymio case, only the wireless version was included (the non-wireless version costs $129 €$ ). For Arduino-based robots, when a specific programming environment is specified, it means that there are programming function templates specific for the platform, which ease programming. Otherwise, the "Arduino-based" designation is used, through the letter A. In the LEGO case, the BOOST ${ }^{5}$ pack, similar to the WeDO, but for the home market, was not considered here, given the classroom focus. In the Fischertechnik case, only the ROBOTICS TXT Discovery Set was considered, since it is the most complete set available. There, however, many other packs are available, ranging from pre-school to high-school, with materials available for specific fields, such as physics, mechanics, etc. The Bot'n Roll One A is a $175 €$ wheeled robotic platform used at RobotParty [18], a several days event where various courses are taught, to allow students to build the robot from parts, including soldering, assembly and programming.

\section{SOFTWARE APPLICATIONS FOR PROGRAMMING ROBOTS}

As important as the robotic platforms themselves, are the materials which allow the teachers and students to effectively used them throughout classes. As such, besides the robotic platforms, we performed a study on the available coding environments and learning resources associated with educational robotic platforms. The study focused only on software applications and/or learning resources which allow connecting to at least one type of physical/real robots, so that students can use one learning environment for programming and interaction with the robots. Programming environments that did not show any activity in 2017 were not considered. A summary of the study can be seen in Table 2, where both coding environments and learning resources were considered.

Table 2 includes, from left to right:

- Project: name and link of the robotic platform;

- Ages: applicable ages;

- Type: can be A if a programming or control applications is included, and $\mathrm{R}$, if learning resources (classes, activities, etc.) are included;

- Programming: types of programming environments included, mainly text-based and visual-based (blocks);

- Internet needed?: indicates whether internet is needed to program the robots. The need for internet can be an obstacle in classroom use;

- Requires install: being able to install an application usually means having administrator privileges, which might not be suitable for classroom use;
- Supported OS: list of operating systems supported. Here, "Web" means that it runs in a browser, thus typically operating system independent;

- Hardware platforms: list of robotic platforms that can be programmed or which there are resources available;

- Teachers training material: whether specific teachers training material is available or not;

- Classroom material: whether classroom material exists and, if so, for which grades. Here " $\mathrm{K}$ " means Kindergarten and NC means that classroom material is available, but is not classified by grade (usually means it is classified by age and/or field);

- Classroom management: whether classroom management is available. A classroom management software is one which allows the teacher to monitor the students' development and manage their assignments;

- Simulation: whether the coding environment includes a simulation. Having a simulator available allows the student to find errors earlier. It also allows the student to develop some work when the robot is not available, or when the number of robots in a class is low. In this last case, the students only tests with the real robot after having done extensive tests with the simulator;

- Registration needed?: whether the students are forced to register using an e-mail. Due to tighter privacy laws in Europe, students should not be required to use their emails to access the learning activities or the coding environments;

- Debug: being able to run the program step by step and evaluate the result can be of great help when evaluating the correctness of a solution.

NQCBaby [19], a language similar with C developed specifically to be more accessible to children, is one of the coding environments often referred in the literature. However, we opted not to include it, since learning resources about this language are not readily available.

Scratch and Blockly are two blocks-based programming environments which are not specific to any robotic platform. However, they are open and include documentation to make them easily adapted to any robotic platform, making them ideal choices as programming environments. Scratch as some advantage due to the number of learning resources available, including classroom specific materials. There are additional coding applications for robots which are based on Scratch or Blockly, which were not included, opting instead to include general information regarding Scratch and Blockly, which also applies to those specific coding applications. There is also Scratch $\mathrm{Jr}^{6}$, which addresses 5 to 7 -year-old children. However, opposite to what happens with Scratch, there are no robotic platforms using ScratchJr. As for Roberta, although it has no specific materials, it can be used with different platforms, and its materials can be adapted to any platform, like Scratch and Blockly. However, although Blockly and Scratch are meant to 
Table 1 - Educational Robots.

\begin{tabular}{|c|c|c|c|c|c|c|c|c|c|c|c|c|c|c|c|c|}
\hline Project & Ages & $\underset{\mathrm{a}}{\text { Type }}$ & \begin{tabular}{|c|}
$\begin{array}{c}\text { Motion } \\
\mathrm{b}\end{array}$ \\
\end{tabular} & $\begin{array}{l}\text { Open- } \\
\text { source }^{\mathrm{c}}\end{array}$ & \begin{tabular}{|c|} 
3D \\
print
\end{tabular} & $\mathbf{T P L}^{\mathrm{d}}$ & VPL ${ }^{\mathrm{e}}$ & $\begin{array}{c}\text { Wirel } \\
\text { comm }^{\mathrm{f}}\end{array}$ & $\begin{array}{c}\text { Prog } \\
\text { upload }^{g}\end{array}$ & Sensors $^{\mathrm{h}}$ & Actuators $^{i}$ & $\begin{array}{c}\text { Brick } \\
\text { compat }\end{array}$ & \begin{tabular}{|c|} 
Learn \\
mat
\end{tabular} & \begin{tabular}{|c|} 
Class \\
mat
\end{tabular} & Use & $\begin{array}{c}\text { Cost } \\
{[€]} \\
\end{array}$ \\
\hline Anprino (Arthur) & N.A. & RKA & $2 \mathrm{~W}$ & Yes & Yes & A & $\mathrm{A}$ & $\mathrm{B}$ & $\mathrm{U}$ & $\mathrm{D}^{\epsilon}, 1^{\epsilon}, \mathrm{a}^{\epsilon}$ & $\mathrm{a}^{\epsilon}, \mathrm{M}$ & No & + & No & - & 70 \\
\hline Bee-Bot & $3+$ & $\mathrm{AP}$ & $2 \mathrm{~W}$ & No & No & No & No & No & $\mathrm{O}$ & No & $\mathrm{M}$ & $\mathrm{No}$ & +++ & ++ & ++++ & 90 \\
\hline Blue-Bot & $5-7$ & $\mathrm{AP}$ & $2 \mathrm{~W}$ & No & No & No & Yes & $\mathrm{B}$ & $\mathrm{B}, \mathrm{O}$ & No & $\mathrm{M}$ & No & +++ & ++ & ++++ & 128 \\
\hline Bot'n Roll ONE A & N.A. & \begin{tabular}{|l|} 
RKA \\
/AP \\
\end{tabular} & $2 \mathrm{~W}$ & S & No & A & $\mathrm{R}$ & No & $\mathrm{U}$ & $\mathrm{D}, \mathrm{B}, \mathrm{a}^{\epsilon}$ & $\mathrm{a}^{\epsilon}, \mathrm{B}, 1, \mathrm{M}, \mathrm{W}$ & No & +++ & No & + & 175 \\
\hline$\frac{\text { BQ Printbot }}{\text { Evolution }}$ & $8+$ & KA & $2 \mathrm{~W}$ & Yes & Yes & $\underline{\mathrm{A}}$ & Yes,A & B & $\mathrm{B}, \mathrm{U}$ & $\mathrm{D}, 1, \mathrm{~L}$ & $\mathrm{~B}, \mathrm{C}$ & No & ++ & + & ++ & 100 \\
\hline BrickPi & $10+$ & KA & $\mathrm{C}$ & $\mathrm{S}$ & No & $\mathrm{P}, \mathrm{J}, \mathrm{N}$ & $\mathrm{S}$ & $\mathrm{W}, \mathrm{B}$ & $\mathrm{W}$ & $1^{\epsilon}, C^{\epsilon}$ & $1, \mathrm{R}$ & Yes & ++ & No & - & 190 \\
\hline Cubelets & $4+$ & KA & $\mathrm{C}$ & No & No & $\mathrm{C}$ & B & B & B & $\mathrm{D}, 1 \mathrm{~L}, \mathrm{~K}^{\epsilon}, \mathrm{T}^{\epsilon}$ & $1, \mathrm{M}, \mathrm{s}$ & Yes & +++ & +++ & +++ & 300 \\
\hline Cubetto & $3+$ & $\mathrm{AP}$ & $2 \mathrm{~W}$ & No & No & $\mathrm{A}$ & $\mathrm{P}, \mathrm{A}$ & $\mathrm{B}$ & $\mathrm{P}$ & $\mathrm{a}$ & $\mathrm{a}^{\epsilon}$ & No & +++ & $++^{\epsilon}$ & ++++ & 219 \\
\hline Cue & $11+$ & $\mathrm{AP}$ & $2 \mathrm{~W}+\mathrm{H}$ & No & No & JS & Yes,B & $\mathrm{B}$ & $\mathrm{B}$ & $\mathrm{A}, \mathrm{D}, \mathrm{S}, \mathrm{B}$ & $\mathrm{E}, 1, \mathrm{M}, \mathrm{S}$ & LEGO & +++ & +++ & +++ & 200 \\
\hline Dash & $6+$ & $\mathrm{AP}$ & $2 \mathrm{~W}+\mathrm{H}$ & No & No & No & Yes,B & $\mathrm{B}$ & $\mathrm{B}$ & $\mathrm{A}, \mathrm{D}, \mathrm{S}, \mathrm{B}$ & $\mathrm{L}, \mathrm{E}, \mathrm{M}, \mathrm{S}$ & LEGO & +++ & +++ & +++ & 150 \\
\hline Edison Robot & $4-16$ & AP & $2 \mathrm{~W}$ & No & No & $\mathrm{P}$ & Yes & No & A & $\mathrm{D}, \mathrm{l}, \mathrm{Ir}, \mathrm{L}$ & $\mathrm{E}, 1, \mathrm{M}$ & LEGO & +++ & +++ & ++ & 50 \\
\hline Finch & $6+$ & AP & $2 \mathrm{~W}$ & $\mathrm{~S}$ & No & $\mathrm{P}, \mathrm{J}, \mathrm{C}, \ldots$ & Yes,S,Sn & No & $\mathrm{U}$ & $\mathrm{D}, \mathrm{L}, \mathrm{t}, \mathrm{A}$ & $\mathrm{L}, \mathrm{M}, \mathrm{S}$ & No & +++ & +++ & ++ & 100 \\
\hline GoPiGo3 & $9+$ & $\mathrm{AP}$ & $2 \mathrm{~W}$ & $\mathrm{~S}$ & No & $\mathrm{P}, \mathrm{N}$ & Yes,S & $\mathrm{W}, \mathrm{B}$ & $\mathrm{W}$ & $\mathrm{D}, \mathrm{f}^{\mathrm{E}}$ & $\mathrm{M}, \mathrm{s}$ & No & ++ & $++^{\epsilon}$ & - & 215 \\
\hline KIBO 18 Kit & $4+$ & KA & $2 \mathrm{~W}$ & No & No & No & $\mathrm{P}$ & No & $\mathrm{P}$ & $\mathrm{D}, \mathrm{S}, \mathrm{L}$ & $1, \mathrm{M}, \mathrm{S}$ & No & ++ & $++^{€}$ & + & 400 \\
\hline \begin{tabular}{|l|} 
LEGO \\
MINDSTORMS EV3 \\
\end{tabular} & $10+$ & KA & $\mathrm{C}$ & No & No & $\begin{array}{l}\text { EB }, C, \\
\text { Java, }, . .\end{array}$ & $\begin{array}{l}\text { Yes, } \underline{\mathrm{S}} \\
\underline{\mathrm{L}}, \ldots\end{array}$ & $\mathrm{W}, \mathrm{B}$ & $\mathrm{B}, \mathrm{U}, \mathrm{W}$ & $\mathrm{D}, \mathrm{B}, \mathrm{R}, \mathrm{Ir}, \mathrm{S}, \mathrm{G}$ & It,l,M,S & LEGO & +++ & +++ & ++ & 400 \\
\hline LEGO WeDo 2.0 & $7+$ & KA & $\mathrm{C}$ & No & No & $\underline{\mathrm{J}}$ & Yes, $\underline{S}$ & $\mathrm{~B}$ & No & $\mathrm{D}, \mathrm{S}, \mathrm{A}, \mathrm{G}, \mathrm{B}$ & $\mathrm{L}, \mathrm{M}, \mathrm{S}$ & LEGO & +++ & +++ & +++ & 175 \\
\hline Makeblock mBot & $8+$ & KA & $2 \mathrm{~W}$ & No & No & $\mathrm{P}$ & $\mathrm{S}$ & IR & $\mathrm{B}, \mathrm{U}, \mathrm{W}$ & $\mathrm{D}, \mathrm{l}, \mathrm{Ir}, \mathrm{L}, \mathrm{A}, \mathrm{G}, \mathrm{B}, \mathrm{K}$ & It,L,M & No & ++++ & ++ & ++ & 99 \\
\hline Marty & $8+$ & KA & $2 \mathrm{~L}$ & $\mathrm{~S}+\mathrm{P}$ & Yes & $\begin{array}{c}\mathrm{P}, \mathrm{N} \\
\mathrm{C}++, \mathrm{C} \#\end{array}$ & $\mathrm{~S}$ & W & $\mathrm{W}$ & A & $\mathrm{s}$ & No & +++ & No & + & 180 \\
\hline Ozobot Bit 2.0 & $6-10$ & $\mathrm{AP}$ & $2 \mathrm{~W}$ & No & No & No & Yes,P & No & $\mathrm{L}$ & $\mathrm{C}, \mathrm{l}, \mathrm{R}$ & $\mathrm{L}, \mathrm{M}$ & No & ++++ & +++ & +++ & 70 \\
\hline Ozobot Evo & $8-14$ & $\mathrm{AP}$ & $2 \mathrm{~W}$ & No & No & No & Yes,P & $\mathrm{B}$ & $\mathrm{L}$ & $\mathrm{C}, \mathrm{D}, \mathrm{R}$ & $\mathrm{L}, \mathrm{M}, \mathrm{S}$ & No & +++ & +++ & ++++ & 102 \\
\hline Phiro Pro & $9+$ & KA & $\mathrm{C}$ & No & No & No & $\begin{array}{l}\text { Yes,S, } \\
\text { Sn,PC }\end{array}$ & B & $\mathrm{B}, \mathrm{O}$ & $\mathrm{D}$ & L,M & LEGO & +++ & ++ & +++ & 212 \\
\hline Pro-Bot & $3+$ & $\mathrm{AP}$ & $2 \mathrm{~W}$ & No & No & $\mathrm{L}$ & Yes & No & $\mathrm{O}, \mathrm{U}$ & $\mathrm{B}, \mathrm{S}, \mathrm{T}, \mathrm{L}$ & $\mathrm{M}, \mathrm{S}, \mathrm{W}$ & Knex & ++ & - & ++ & 139 \\
\hline $\begin{array}{l}\text { ROBOTICS TXT } \\
\text { Discovery Set } \\
\end{array}$ & $10+$ & KA & $\mathrm{C}$ & No & No & $\underline{P}$ & Yes & $\mathrm{W}, \mathrm{B}$ & $\mathrm{U}$ & $\mathrm{C}, \mathrm{D}, \mathrm{t}, \mathrm{B}$ & $\mathrm{D}, \mathrm{E}, \mathrm{M}, \mathrm{S}$ & No & ++ & $++^{\epsilon}$ & + & 400 \\
\hline Sphero SPRK+ & $8+$ & $\mathrm{AP}$ & $2 \mathrm{~W}$ & No & No & JS & Yes & $\mathrm{B}$ & $\mathrm{B}$ & $\mathrm{A}, \mathrm{G}$ & 1,L,M,S & No & +++ & +++ & +++ & 150 \\
\hline $\begin{array}{l}\text { STEM ROBOTICS } \\
\text { ERP PRO } 1.3 \\
\end{array}$ & $8+$ & KA & $\mathrm{C}$ & No & No & Yes & Yes & B & $\mathrm{B}, \mathrm{U}$ & $\mathrm{D}, \mathrm{T}$ & $\mathrm{B}, 1, \mathrm{M}$ & $\begin{array}{c}\text { Engino } \\
\text { toys }\end{array}$ & +++ & ++ & ++ & 250 \\
\hline Thymio II wireless & $6+$ & $\mathrm{AP}$ & $2 \mathrm{~W}$ & Yes & Yes & Yes & Yes,B, $\underline{\mathrm{S}}$ & Yes & Yes,U & $\mathrm{A}, \mathrm{B}, \mathrm{D}, \mathrm{Ir}, 1, \mathrm{~S}, \mathrm{t}$, & $\mathrm{L}, \mathrm{M}, \mathrm{S}$ & LEGO & +++ & ++ & +++ & 189 \\
\hline VEX IQ Super Kit & $8+$ & KA & $\mathrm{C}$ & No & No & eC,P & Yes,S,B & Yes & $\mathrm{U}$ & $\mathrm{B}, \mathrm{D}, \mathrm{R}, \mathrm{T}$ & $1, \mathrm{M}$ & No & $+++^{\epsilon}$ & $+++^{\epsilon}$ & ++ & 330 \\
\hline Zowi & $8+$ & $\mathrm{AP}$ & $2 \mathrm{~L}$ & Yes & Yes & $\underline{\mathrm{A}}$ & Yes,A & $\mathrm{B}$ & $\mathrm{B}, \mathrm{U}$ & $\mathrm{D}, \mathrm{S}$ & $\mathrm{B}, 1, \mathrm{~s}$ & No & +++ & ++ & +++ & 130 \\
\hline
\end{tabular}

a. RKA - Raw Kit Assembly; KA - Kit Assembly; AP - Assembled Platform. ${ }^{\text {b. }} \mathrm{xW}$ - Motion based on $\mathrm{x}$ wheels; H - Head motion; C - Configurable. ${ }^{\mathrm{c}} \mathrm{S}-\mathrm{Schematic}$ only; P - 3D parts. ${ }^{\mathrm{d}} \mathrm{A}-$ Arduino-based; EB - EV3Basic; eC - easyC; J - Java; JS - Javascript; L - LOGO; N - NodeJS; P - Python; Pr - Processing; Yes - Manufacturer specific. ${ }^{\text {e }}$ A - Arduino-based; B - Blockly (or blockly-based); L - LabView; P - Physical programming; PC - Pocket Code; R - Open Roberta; S - Scratch (or Scratch-based); Sn - Snap; Yes - manufacturer specific. ${ }^{\text {ff }}$ B - Bluetooth; W -

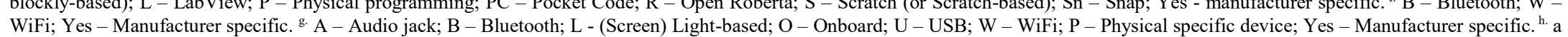
- Any sensor; A - Accelerometer (1,2 or 3 axis); B - Buttons or bumper switches; C - Camera; D - Distance or proximity sensors; G - Gyroscope (1, 2 or 3 axis); Ir - Infrared receiver; K Knob; 1 - Line sensor (infrared or color); L - Light sensors; R - Single color sensor; S - Sound sensor; $t$ - Temperature sensor; T - Touch sensor. ${ }^{i}$ a - Any; B - Buzzer/Piezo; C - Continuous Knob; - Line sensor (infrared or color); L - Light sensors; R - Single color sensor; S - Sound sensor; t - Temperature sensor; T - Touch sensor. ${ }^{1}$ a - Any; B - Buzzer/Piezo; C - Continuous
rotations servos; D - Color display; E - Encoders; It - Infrared transmitter; 1 - Single color LEDs or lights; L - Color LEDs; M - Motors; R - uSD card reader; s - Servomotors; S - Sound (speaker); $\mathrm{W}$ - Single-color display: ${ }^{\epsilon}$ - With an additional cost.

The project name links to its website, and “...” mean additional options available but not included, due to space reasons. Underscore items means $3^{\text {rd }}$ party source. N.A. means Not Available. 
Table 2 - Educational robotics software and resources.

\begin{tabular}{|c|c|c|c|c|c|c|c|c|c|c|c|c|c|c|}
\hline Project & Ages & Type $^{\mathrm{d}}$ & Lang & Programming & $\begin{array}{l}\text { Internet } \\
\text { needed? }\end{array}$ & $\begin{array}{c}\text { Requires } \\
\text { install } \\
\end{array}$ & $\begin{array}{l}\text { Supported } \\
\text { OS } \\
\end{array}$ & $\begin{array}{l}\text { Hardware } \\
\text { platforms }\end{array}$ & $\begin{array}{c}\text { Teachers train } \\
\text { material }\end{array}$ & $\begin{array}{c}\text { Class } \\
\text { material }\end{array}$ & $\begin{array}{c}\text { Class } \\
\text { manag }\end{array}$ & $\begin{array}{c}\text { Simula } \\
\text { tion }\end{array}$ & $\begin{array}{c}\text { Reg } \\
\text { needed? }\end{array}$ & Debug \\
\hline$\frac{\text { Arduino }}{\text { Education }}$ & N.A. & $A, R$ & en & $\begin{array}{c}\text { Visual } \\
\text { (Ardublockly, } \\
\text { S4A })^{\text {b }} \text { Text } \\
(\mathrm{C} / \mathrm{C}++) \\
\end{array}$ & No & $\begin{array}{c}\text { Yes } \\
\text { (offline } \\
\text { version) }\end{array}$ & $\begin{array}{l}\text { Linux, Mac, } \\
\text { Windows }\end{array}$ & Arduino(-based) & Yes $^{b}$ & $13-17^{\mathrm{b}, \epsilon}$ & Yes & No & Yes & No \\
\hline$\underline{\text { Aseba }}$ & $6+$ & $\mathrm{A}, \mathrm{R}$ & $\begin{array}{c}\text { en, fr, de, es, } \\
\text { it, nl, zh }\end{array}$ & $\begin{array}{c}\text { Visual (VLP, } \\
\text { Blockly, Scratch), } \\
\text { Text (Aseba) }\end{array}$ & No & Yes & $\begin{array}{l}\text { Linux, Mac, } \\
\text { Windows }\end{array}$ & Thymio & Yes $^{\mathrm{a}}$ & $1-6^{a}$ & No & Yes & No & Yes \\
\hline$\underline{\text { Blockly }}$ & $4+$ & A & $44+$ & Visual (Blockly) & Yes & $\begin{array}{c}\text { Yes } \\
\text { (Android, } \\
\text { iOS) }\end{array}$ & $\begin{array}{c}\text { Android, iOS, } \\
\text { Web }\end{array}$ & $\begin{array}{c}\text { Cubelets, Cue, Dash, } \\
\text { Dot, Ozobot Bit 2, } \\
\text { Ozobot Evo, Thymio, } \\
\text { Vex IQ } \\
\end{array}$ & No & No & No & No & No & No \\
\hline$\underline{\text { CREATE }}$ & $6+$ & $\mathrm{A}, \mathrm{R}$ & en & $\begin{array}{l}\text { Visual (Scratch, } \\
\text { Snap }\end{array}$ & No & Yes & $\begin{array}{l}\text { Linux, Mac, } \\
\text { Windows }\end{array}$ & Finch & Yes & $\mathrm{K}-12$ & No & No & No & No \\
\hline Edison & $4+$ & $\mathrm{A}, \mathrm{R}$ & en & \begin{tabular}{|c|} 
Visual (EdBlocks), \\
Hybrid (EdWare), \\
Text (EdPy) \\
\end{tabular} & Yes & No & Web & Edison & Yes & $3-7+$ & No & No & No & No \\
\hline$\underline{\text { Fischertechnik }}$ & & $\mathrm{A}, \mathrm{R}$ & $\begin{array}{c}\text { de, en, fr, nl, } \\
\text { es, pt }\end{array}$ & $\begin{array}{c}\text { Visual (ROBO Pro, } \\
\text { Brickly) }\end{array}$ & No & Yes & $\begin{array}{l}\text { Android, iOS, } \\
\text { Windows }\end{array}$ & Fischertechnik & Yes & $\begin{array}{l}2-12 \\
(\mathrm{NC})\end{array}$ & No & No & No & No \\
\hline$\frac{\underline{\text { LEGO }}}{\text { Education }}$ & $3+$ & $\mathrm{A}, \mathrm{R}$ & \begin{tabular}{|c|} 
zh, de, en, ja, \\
ko, ru, ar, da, \\
es, fr, no, nl, \\
pt
\end{tabular} & $\begin{array}{l}\text { Visual (EV3, } \\
\text { WeDo) }\end{array}$ & No & Yes & $\begin{array}{c}\text { Android, } \\
\text { Chromebook, } \\
\text { iOs, Mac, } \\
\text { Windows } \\
\end{array}$ & $\begin{array}{l}\text { LEGO WeDO, LEGO } \\
\text { Mindstorms }\end{array}$ & Yes $^{\mathrm{a}}$ & $1-8^{\mathrm{a}, \mathrm{b}}$ & No & $\begin{array}{l}\text { Yes } \\
\text { (build } \\
\text { model } \\
\text { only) } \\
\end{array}$ & No & No \\
\hline$\underline{\text { MakeCode }}$ & N.A. & $\mathrm{A}, \mathrm{R}$ & $\begin{array}{c}\text { zh, de, en, fr, } \\
\text { it, ja, ko, si, } \\
\text { nl, no, sv }\end{array}$ & $\begin{array}{l}\text { Visual (Blockly), } \\
\text { Text (JavaScript) }\end{array}$ & Yes & $\mathrm{Yes}^{\mathrm{c}}$ & Web & $\begin{array}{c}\text { micro:bit, circuit } \\
\text { playground, Chibi } \\
\text { Chip, Grove Zero, } \\
\text { Sparkfun Inventors Kit }\end{array}$ & $\begin{array}{c}\text { Yes (micro:bit, } \\
\text { Circuit } \\
\text { Playground, } \\
\text { minecraft) }\end{array}$ & $6-8$ & No & Yes & No & No \\
\hline$\underline{\text { Roberta }}$ & N.A. & A & \begin{tabular}{|c|} 
cs, da, de, \\
en, es, fi, fr, \\
it, nl, pl, pt, \\
ru, tr
\end{tabular} & Visual (NEPO) & Yes & No & Web & $\begin{array}{l}\text { EV3, NXT, micro:bit, } \\
\text { Bot'n Roll, NAO, } \\
\text { BOB3, Calliope }\end{array}$ & No & No & No & Yes & No & No \\
\hline$\underline{\text { Scratch }}$ & $8-16$ & $\mathrm{~A}, \mathrm{R}$ & $40+($ App $)$ & Visual (Scratch) & $\begin{array}{c}\text { No } \\
\text { (offline } \\
\text { version) }\end{array}$ & $\begin{array}{c}\text { Yes } \\
\text { (offline } \\
\text { version) }\end{array}$ & $\begin{array}{l}\text { Linux, Mac, } \\
\text { Windows, } \\
\text { Web }\end{array}$ & $\begin{array}{c}\text { BrickPi, Finch, WeDo, } \\
\text { mBot, EV3, GoPiGo3, } \\
\text { Marty, Phiro, Thymio, } \\
\text { Vex IQ Super Kit }\end{array}$ & Yes & $\begin{array}{l}\mathrm{K}-12 \\
(\mathrm{NC})\end{array}$ & Yes & Yes & Yes & No \\
\hline$\underline{\text { Tynker }}$ & $6+$ & $\mathrm{A}, \mathrm{R}$ & en & $\begin{array}{l}\text { Visual (blocks), } \\
\text { Text (Javascript, } \\
\text { Python, Swift) }\end{array}$ & Yes & No & Web & $\begin{array}{l}\text { Parrot drones, LEGO } \\
\text { WeDo, Sphero, Ollie }\end{array}$ & Yes $^{\epsilon}$ & $\mathrm{K}-8^{€}$ & $\mathrm{Yes}^{\epsilon}$ & Yes & Yes & N.A. \\
\hline$\underline{\text { Wonder }}$ & $4+$ & $\mathrm{R}$ & $\mathrm{en}, \mathrm{pt}^{\mathrm{b}}$ & $\begin{array}{c}\text { Visual (Blockly, Go, } \\
\text { Path, Xylo,Wonder), } \\
\text { Physical (Puzzlets) }\end{array}$ & No & Yes & Android, iOS & Dash \& Dot & Yes & $K-5^{\epsilon}$ & No & No & Yes & No \\
\hline
\end{tabular}

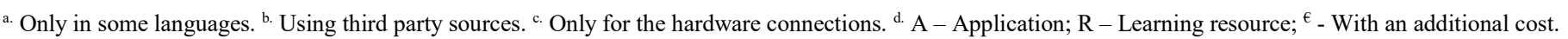

The project name links to its website due to space reasons. 
be adapted, meaning that the user as several different Blockly or Scratch-based applications, one per different robotic platform, Roberta aims at using a centralized point of entry for different platforms with an easier adaptation for different platforms. This can be useful for BYOD (Bring Your Own Devices) classes, or for faster introduction of a new robotic platform. Furthermore, Roberta includes a simulation engine, which can greatly reduce the trial and error time, particularly at a starting stage of a new project. Similarly, MakerCode, provides a coding and simulation environment for different platforms. However, MakerCode works at a lower-level, closer to the electronic boards that control the robots. This means that, considering educational robots, MakerCode can be more effective at a later stage, when the students start working directly with these electronic boards.

Regarding the age property in Table 2, it is interesting to compare with some ages from Table 1, for a given robot. The ages given by the learning material suppliers is usually lower than the age provided by the robot manufacturers, given the implicit presence of the teacher and the learning resources.

\section{CONCLUSIONS AND FUTURE WORK}

Even though the number of educational robotic platforms and associated learning resources is increasing, literature review shows that LEGO based robotic activities (using the RCX, NXT or EV3 kits) are still dominating the market (around 90\%, according to [8]). Table 1 only included robotic platforms which have one or more pre-defined robot models that the user can build or use pre-assembled. There are currently many materials related with physical computing which, together with learning materials, also provide excellent hands-based learning tools for STEAM development. These include, among others, the Arduino $^{7}$, micro:bit ${ }^{8}$ and Calliope ${ }^{9}$ ecosystems. The reason to approach only robotic-based (almost) ready platforms was to decrease the learning curve and provide for a richer experience in K-12 grades.

The goal of this project was not so much as to provide conclusions on how to use robotic platforms for STEAM development, but mostly to provide the needed information for a prospect user to make a knowledge-based decision. In that sense, several key issues were also discussed and evaluated in the study.

For future work, the full dataset containing the information presented here will be publicly available online and will be open for controlled updates by any user, together with links for learning materials. This centralized source of information and learning indications can be used as a starting point for any teacher working with or developing educational robotics. On a final note, please beware most of the information provided here is based in the documentation provided by the manufacturers, and not from a first person hands-on experiments.

\section{REFERENCES}

[1] T. Hohlfeld, A. Ritzhaupt, K. Dawson and M. Wilson, "An examination of seven years of technology integration in Florida schools: Through the lens of the Levels of Digital Divide in Schools," Computers \& Education, vol. 113, pp. 135 - 161, 2017.

[2] A. Blignaut, J. Hinostroza, C. Els and M. Brun, "ICT in education policy and practice in developing countries: South Africa and Chile compared through SITES 2006," Computers \& Education, vol. 55, no. 4, pp. 1552 - 1563, 2010.

[3] E. Commission/EACEA, "Developing Key Competences at School in Europe: Challenges and Opportunities for Policy," Eurydice, Luxembourg, 2012.

[4] S. Grover and R. Pea, "Computational Thinking in K-12: A Review of the State of the Field," Educational Researcher, vol. 42, no. 1, pp. 38-43, 2013.

[5] M. Bers, I. Ponte, C. Juelich, A. Viera and J. Schenker, "Teachers as Designers: Integrating Robotics in Early Childhood Education," Information Technology in Childhood Education Annual, vol. 2002, no. 1 , pp. 123 - 145, 2002.

[6] A. Eguchi, "Bringing Robotics in Classrooms," in Robotics in STEM Education: Redesigning the Learning Experience, Springer International Publishing, 2017, pp. 3 - 31.

[7] M. Lieto, E. Inguaggiato, E. Castro, F. Cecchi, G. Cioni, M. Omo, C. Laschi, C. Pecini, G. Santerini, G. Sgandurra and P. Dario, "Educational Robotics intervention on Executive Functions in preschool children: A pilot study," Computers in Human Behavior, vol. 71, pp. 16 - 23, 2017.

[8] F. Barreto and V. Benitti, "Exploring the educational potential of robotics in schools: A systematic review," Computers \& Education, vol. 58, no. 3, pp. 978 - 988, 2012.

[9] O. Mubin, C. Stevens, S. Shahid, A. Mahmud and J. Dong, "A Review of the Applicability of Robots in Education," Technology for Education and Learning, vol. 1, no. 2013, 2013.

[10] P. Seymour, Mindstorms: Children computers and powerful ideas, New York: Basic Books Inc., 1980.

[11] L. Gerber, A. Calasanz-Kaiser, L. Hyman, K. Voitiuk, U. Patil and I. Riedel-Kruse, "Liquid-handling Lego robots and experiments for STEM education and research," PLOS Biology, vol. 15, no. 3, pp. 1-9, 2017.

[12] D. Alimisis, "Educational robotics: Open questions and new challenges," Academic Journal, vol. 6, no. 1, pp. 63 - 71, 2013.

[13] B. Barker and J. Ansorge, "Robotics as Means to Increase Achievement Scores in an Informal Learning Environment," Journal of Research on Technology in Education, vol. 39, no. 3, pp. 229 - 243, 2007.

[14] C. Kim, D. Kim, J. Yuan, R. Hill, P. Doshi and C. Thai, "Robotics to promote elementary education pre-service teachers' STEM engagement, learning, and teaching," Computers \& Education, vol. 91, pp. 14 - 31, 2015.

[15] M. Karim, S. Lemaignan and F. Mondada, "A review: Can robots reshape K-12 STEM education?," in IEEE International Workshop on Advanced Robotics and its Social Impacts (ARSO), Lyon, France, 2015.

[16] F. García-Peñalvo, A. Rees, J. Hughes, I. Jormanainen, T. Toivonen and J. Vermeersch, "A Survey of Resources for Introducing Coding into Schools," in Proceedings of the Fourth International Conference on Technological Ecosystems for Enhancing Multiculturality, Salamanca, Spain, 2016.

[17] A. Özgür, S. Lemaignan, W. Johal, M. Beltran, M. Briod, L. Pereyre, F. Mondada and P. Dillenbourg, "Cellulo: Versatile Handheld Robots for Education," in Proceedings of the 2017 ACM/IEEE International Conference on Human-Robot Interaction, Vienna, Austria, 2017.

[18] F. Ribeiro, G. Lopes, N. Pereira and J. Cruz, "Learning Robotics for Youngsters - The RoboParty Experience," in Robot 2015: Second Iberian Robotics Conference, Lisbon, Portugal, 2016.

[19] G. Demo, "Programming Robots in Primary Schools Deserves a Renewed Attention," in Emerging Technologies and Information Systems for the Knowledge Society, Berlin, Heidelberg, 2008. 